\title{
Fenofibrate Increases Radiosensitivity in Head and Neck Squamous Cell Carcinoma via Inducing G2/M Arrest and Apoptosis
}

\author{
Jia Liu ${ }^{1 \&}$, Yang-Yang Ge ${ }^{3 \&}$, Hong-Cheng Zhu ${ }^{1 \&}$, Xi Yang ${ }^{1}$, Jing Cai ${ }^{3}$, Chi Zhang ${ }^{1}$, \\ Jing Lu ${ }^{1}$, Liang-Liang Zhan ${ }^{1}$, Qin Qin ${ }^{1}$, Yan Yang ${ }^{1}$, Yue-Hua Yang ${ }^{1}$, Hao Zhang ${ }^{1}$, \\ Xiao-Chen Chen ${ }^{1}$, Zhe-Ming Liu ${ }^{1}$, Jian-Xin $\mathrm{Ma}^{4}$, Hong-Yan Cheng ${ }^{2}$, Xin-Chen Sun ${ }^{1 *}$
}

\begin{abstract}
Radiation therapy is an important treatment for head and neck squamous cell carcinoma (HNSCC). However, how to promote radiation sensitivity in HNSCC remains a challenge. This study aimed to investigate the radiosensitizing effects of fenofibrate on HNSCC and explore the underlying mechanisms. HNSCC cell lines CNE-2 and KB were subjected to ionizing radiation (IR), in the presence or absence of fenofibrate treatment. Cell growth and survival, apoptosis and cell cycle were evaluated. In addition, $\mathrm{CNE}-2$ cells were xenografted into nude mice and subjected to IR and/ or fenofibrate treatment. The expression of cyclinB and CDK1 was detected by Western blotting. Our results showed that fenofibrate efficiently radiosensitized HNSCC cells and xenografts in mice, and induced apoptosis and G2/M arrest via reducing the activity of the CDK1/cyclinB1 kinase complex. These data suggest that fenofibrate could be a promising radiosensitizer for HNSCC radiotherapy.
\end{abstract}

Keywords: Fenofibrate - HNSCC - cell cycle - radiotherapy

Asian Pac J Cancer Prev, 15 (16), 6649-6655

\section{Introduction}

Head and neck cancers include malignancies of the oral cavity, nasopharynx, oropharynx, hypopharynx, larynx, paranasal sinuses and salivary glands. Squamous cell carcinoma is the most common histological type of head and neck cancer, accounting for $90 \%$ of all head and neck malignancies. Head and neck squamous cell carcinoma (HNSCC) is the sixth most common malignancy worldwide (Parkin et al., 2005). Despite the predominating primary therapy of radiation (RT), chemoradiation (CRT) or surgery, a 5-year survival rate is not obviously improved as a result of local recurrence and metastases (Ragin et al., 2007; Li et al., 2013). Ionizing radiation is one of the most effective approaches for local control. However, current standard chemoradiation strategies have reached the upper limits of toxicity with complications and side effects, consequently preventing successful treatment. Therefore, the problem of how to increase tumor response to irradiation with targeted sensitizers has become a research focus in radiotherapy of HNSCC (Akervall et al., 2014; Zhang et al., 2014).

Cellular proliferation is regulated primarily by the regulation of cell cycle, which consists of four distinct sequential phases (G0/G1, S, G2 and M). In eukaryotes, the cell cycle is regulated by cyclins, cyclin-dependent kinases (CDKs) and cyclin-dependent kinase inhibitor (CDKI). In particular, cyclin B and CDK1 proteins are involved in regulating the progression of $\mathrm{G} 2 / \mathrm{M}$ phase (Cho et al., 2013). It is widely known that cells are blocked in the G2/M phase during DNA damage, and cells are more susceptible to the cytotoxic effects of radiotherapy in the G2/M phase (Pawlik et al., 2004). Increasing irradiationinduced G2/M phase arrest allows cell death may be a strategy being employed in cancer therapeutics (Hematulin et al., 2012; Cui et al., 2012).

Fenofibrate are ligands for peroxisome proliferatoractivated receptor $\alpha$ and have been associated with the treatment of diabetes, hyperlipidemia and cardiovascular diseases for many years, as they modulate the expression of genes regulating lipid and glucose metabolism (Balfour et al., 1990). Interestingly, more recent studies suggest that fenofibrate may have an important role as antitumor agents in various cancers such as glioblastoma (Wilk et al., 2012), prostate cancer (Zhao et al., 2013), lung cancer (Liang et al., 2013), breast cancer (Li et al., 2014), endometrial cancer (Saidi et al., 2006), colon cancer (Watanabe et al., 2013), melanoma (Grabacka 
et al., 2006), medulloblastoma (Urbanska et al., 2008) and leukemia (Zak et al., 2010). Fenofibrate decreases metastatic potential of melanoma cells in vitro via downregulation of Akt (Grabacka et al., 2006). Fenofibrate induced apoptosis and decreased proliferation rate in endometrial cancer cells (Saidi et al., 2006), in human and mouse medulloblastoma cell lines (Urbanska et al., 2008), in mantle cell lymphoma (Zak et al., 2010), and in triple-negative breast cancer cells (Li et al., 2014). In addition, Fenofibrate, also enhanced sensitivity to 5-FU with dephosphorylated ERK $1 / 2$ and a reduction of thymidylate synthase (TS) expression on human colon cancer cells (Watanabe et al., 2013). Fenofibrate-induced nuclear translocation of FoxO3A triggers Bim-mediated apoptosis in glioblastoma cells in vitro (Wilk et al., 2012). It was also reported that fenofibrate suppressed cell proliferation by regulating the cell cycle such as inducing G0/G1 arrest (Wilk et al., 2012; Zhao et al., 2013; Liang et al., 2013; Watanabe et al., 2013; Li et al., 2014) or G2/M arrest (Urbanska et al., 2008; Joe et al., 2010). In spite of these multiple observations, molecular mechanisms by which fenofibrate targets cancer cells are not well defined.

However, the potential effects of fenofibrate on the radiosensitivity of HNSCC and the underlying mechanism have not been reported. In this study, the change in radiosensitivity of oral epidermoid carcinoma cells and nasopharyngeal carcinoma cells after fenofibrate treatment was investigated, and the potential mechanism was also explored in vivo and in vitro, to provide theoretical basis for the radiotherapy of HNSCC.

\section{Materials and Methods}

\section{Cell culture, Irradiation and Reagents}

Human oral epidermoid carcinoma cell KB and nasopharyngeal carcinoma cell CNE-2 was obtained from Shanghai Institute of Cell Biology (Shanghai, China). Both cell types were cultured in Dulbecco's modified Eagle's medium (DMEM; Gibco), supplemented with $10 \%$ fetal bovine serum (Hyclone) and $1 \%$ penicillin/streptomycin (Invitrogen). Irradiation group of cells were subjected to a 2, 4, 6, 8 Gy X-ray irradiation from a medical linear accelerator (Elekta Precise, Stockholm, Sweden) at room temperature. Fenofibrate was purchased from Sigma and dissolved in dimethyl sulfoxide (DMSO) as a stock solution of $200 \mathrm{mM}$. Anti-cyclin B1 mouse antibody, antiCDK1 mouse antibody, anti- $\beta$-actin antibody, anti-mouse antibody and anti-rabbit antibody were purchased from Santa Cruz Biotechnology.

\section{Cell viability assay}

The cell proliferation rate was estimated by using CCK8. 5000 cells/well were seeded into 96-well plates and treated with different concentrations of fenofibrate. After 24 and 48 hours, $10 \mu \mathrm{l} \mathrm{CCK}-8$ reagent was added to each well. After 2 hours incubation at $37^{\circ} \mathrm{C}$ in humidified $5 \% \mathrm{CO} 2$, plates were read on a microplate reader. Relative cell viability of an individual sample was calculated by normalizing their absorbance to that of the corresponding control.

\section{Clonogenic survival assay}

CNE-2 and KB cells were trypsinized as single-cell suspension and seeded into 60-mm dishes. After cell adhesion, they were treated with DMSO (control) or fenofibrate $(50 \mu \mathrm{M}$ or $100 \mu \mathrm{M})$ for $24 \mathrm{~h}$, and then exposed to different doses of radiation $(0,2,4,6$ or $8 \mathrm{~Gy})$. After 14 days, the cells were fixed with $70 \%$ ethanol and stained with Giemsa. The survival enhancement ratio (SER) was calculated as the ratio of the mean inactivation dose in control cells divided by the mean inactivation dose in fenofibrate-treated cells.

\section{Apoptosis assay}

Annexin V-FITC and propidium iodide dual staining was performed to assess the fraction of apoptotic cells. The cells were seeded into six-well plates and treated with or without fenofibrate in normoxic conditions for 24 hours, then subjected to X-ray irradiation (8 Gy). Twenty-four hours after radiation, the cells were collected and analyzed using an Annexin V-FITC Apoptosis Detection kit (BD Bioscience, Oxford, UK) in the Flow Cytometry.

\section{Measurement of caspase-3 activity}

The activity of caspase- 3 was determined using the caspase- 3 activity kit (Beyotime Institute of Biotechnology, China). To evaluate the activity of caspase-3, cell lysates were prepared after their respective treatment with various designated treatments. Assays were performed on 96well microtitre plates by incubating $10 \mu \mathrm{L}$ protein of cell lysate per sample in $80 \mu \mathrm{L}$ reaction buffer $(1 \% \mathrm{NP}-40,20$ $\mathrm{mmol} / \mathrm{L}$ Tris- $\mathrm{HCl}(\mathrm{pH} 7.5), 137 \mathrm{mmol} / \mathrm{L} \mathrm{NaCl}$, and $10 \%$ glycerol $)$ containing $10 \mu \mathrm{L}$ caspase- 3 substrate $(2 \mathrm{mmol} / \mathrm{L}$ Ac-DEVD-pNA). The lysates were incubated at $37^{\circ} \mathrm{C}$ for 4 hours. The samples were measured with a microplate reader at an absorbance of $405 \mathrm{~nm}$. The detailed analysis procedure was according to the manufacturer's protocol. The caspase- 3 activities were expressed by comparing with the results of the controls.

\section{Cell cycle analysis}

The cells in exponential growth phase were trypsinized with $0.25 \%$ trypsin and a single-cell suspension was used. And then cells were seeded in six-well plates at a density of $20 * 10^{\wedge} 4$ cells/well and allowed to adhere for 24 hours. And then he cells were treated with or without fenofibrate in normoxic conditions for 24 hours ( 50 and $100 \mu \mathrm{mol} / \mathrm{L}$ ) and then irradiated at a dose of $4 \mathrm{~Gy}$. Subsequently, the cells were trypsinized at 24 hours after irradiation. Cells from each group were harvested and washed with PBS. Cold ethanol (70\%) was added and then stored at $-20^{\circ} \mathrm{C}$ overnight. Cells were stained with PI treated with RNase A $(5 \mu \mathrm{g} / \mathrm{mL})$ and subjected to flow cytometry (FACSCalibur) for cell cycle analysis.

\section{Western blot analysis}

Cells were harvested and homogenized in RIPA lysis buffer and centrifuged at 12,000 rpm for 20 minutes at $4{ }^{\circ} \mathrm{C}$. Protein concentrations of the supernatants were determined using BCA assay. Equal amounts of protein were loaded into each well and separated by $10 \%$ sodium dodecyl sulfate polyacrylamide gel 
electrophoresis and then blotted onto PVDF membranes. The membranes were blocked and then probed with primary antibodies at $4^{\circ} \mathrm{C}$ overnight followed by incubated with conjugated secondary antibodies for 1 hour at $37^{\circ} \mathrm{C}$. Immunoblotted proteins were visualized by ECL reagents and the signals were detected by Chemidoc XRS imaging system (Quantity One Quantitation software, BioRad Laboratories, Hercules, CA, USA).

\section{Xenograft mouse model}

Animal experiments were approved by the Ethics Committee of Nanjing Medical University. Male BALB/C nude mice (16-22 g, 4-5 weeks old) were provided by Nanjing Medical University Animal Center. CNE-2 cells were suspended in PBS and injected into the right axilla of nude mice $\left(1-5^{*} 10^{\wedge} 6\right.$ cells per animal) subcutaneously. When tumor volume increased to $100-300 \mathrm{~mm}^{3}$, the animals were randomly grouped into four different groups ( $\mathrm{n}=6$ ); (1) vehicle (PBS), (2) 8Gy irradiation, (3) $10 \mathrm{mg} / \mathrm{kg}$ fenofibrate, (4) $10 \mathrm{mg} / \mathrm{kg}$ fenofibrate plus $8 \mathrm{~Gy}$ irradiation. The mice in the control group were treated with vehicle control, whereas the mice in groups 3 and 4 were given daily intraperitoneal injection of $10 \mathrm{mg} / \mathrm{kg}$ fenofibrate on day 9 and day 10. Tumors were irradiated using an RS-2000 biological irradiator at a dose of 8 Gy with X-rays ( $2 \mathrm{~Gy} / \mathrm{min})$ delivered 2 hours after injection on day 10. Tumor growth was measured every 2 days and the tumor volume was calculated according to the formula: tumor volume $=\left(\right.$ length $(\mathrm{L})^{*}$ width $\left.(\mathrm{W})^{2}\right) / 2$. The tumor doubling time (DT) was calculated as follows: $\mathrm{DT}=\mathrm{d}^{*} \lg 2 / \lg \left(\mathrm{V}_{\mathrm{d}} / \mathrm{V}_{0}\right)$, where $\mathrm{d}$ was the length of time between two measurements, $\mathrm{Vd}$ was the volume of the tumor treated with $\mathrm{X}$-ray, and $\mathrm{V}_{0}$ was the volume of the tumor before the $\mathrm{X}$-ray. The mice were sacrificed till day 24 and during the period, no mouse died. Tumor tissues were completely dissected, weighed, and then stored in $4 \%$ buffered formaldehyde.
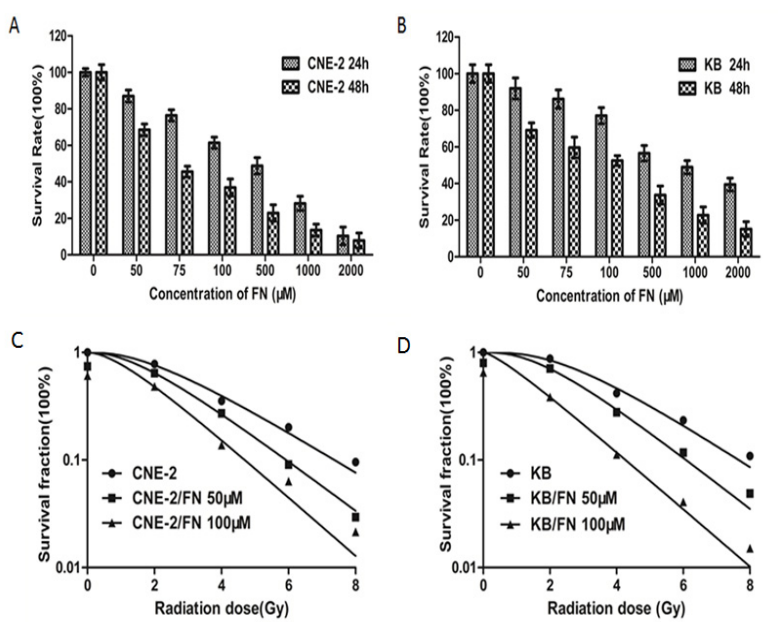

Figure 1. Fenofibrate Inhibited Growth of HNSCC Cells in a Time- and Dose-Dependent Manner ( $A$ and B). Clonogenic assay showing that fenofibrate sensitized HNSCC cells to IR significantly in normoxia environment and there was a dose-dependent reduction $(\mathrm{C}$ and $\mathrm{D}) . \mathbf{A}$ : CNE-2 cell lines, B: KB cell lines. C: CNE-2 cell lines, D: KB cell lines. FN: fenofibrate.

\section{Statistical analysis}

Mean \pm SE from triplicate assays were calculated and the differences between treatment groups were determined using a two-tailed Student's t test. Statistical analysis was performed using Prism 5.0 software (GraphPad Prism). $p<0.05$ was considered statistically significant.

\section{Results}

Fenofibrate significantly induced apoptosis and enhance radiosensivity of both $\mathrm{CNE}-2$ and $\mathrm{KB}$ cell lines

In the first experiment, cell viability was determined using a CCK8 assay. We found that fenofibrate treatment inhibited the growth of CNE-2 and KB cell lines in a time and dose dependent manner (Figure 1A and 1B). At 24 hours, the IC50 (half maximal inhibitory concentration) for HNSCC cells CNE-2 and KB was $177.96 \mu \mathrm{M}$ and $1170.22 \mu \mathrm{M}$ respectively. Thus we treated cells for 24 hours with a safe concentration of fenofibrate $(50 \mu \mathrm{M}$ and $100 \mu \mathrm{M}$ ) for the following experiments.

To investigate the effects of fenofibrate on radiotherapy sensitization of HNSCC cells, we performed a clonogenic assay and found fenofibrate sensitized HNSCC cells to IR significantly in normoxia environment. The cells were treated by fenofibrate for 24 hours at $50 \mu \mathrm{M}$ and 100 $\mu \mathrm{M}$, then irradiated with doses of 0-8 Gy, and plated to evaluate the colony-forming efficiency. Untreated cells were used as the control. After 14 days, the colonies were counted, and the survival curves of the cells treated with fenofibrate and irradiation were constructed compared with those of irradiation alone. In response to radiation, there was a dose-dependent reduction in cell survival with or without fenofibrate treatment. The dose-survival curves were shown in Figure 1C. The SF data were fitted into the single hit multi target model formula: $S F=1-\left(1-\mathrm{e}^{-\mathrm{D} / \mathrm{D} 0}\right)^{\mathrm{n}}$. The results showed that the survival fraction $\left(\mathrm{SF}_{2}\right)$ at 2 Gy was 0.76 for CNE- 2 cells, and 0.84 for KB cells, respectively. After treatment with Fenofibrate with $50 \mu \mathrm{M}$ and $100 \mu \mathrm{M}, \mathrm{SF}_{2}$ of cells decreased to 0.64 and 0.48 in CNE- 2 cells, and 0.70 and 0.38 in KB cells, respectively. The sensitizing enhancement ratio $\left(\mathrm{SER}_{\mathrm{D} 0}\right)$ was 1.21 and 1.42 for CNE-2, and 1.19 and 1.32 for KB cells (Table 1 and 2). These observations indicated that treating HNSCC cells with Fenofibrate resulted in a significant radiosensitization effect.

Next we detected the effects of apoptosis of CNE-2 cells treated by fenofibrate, irradiation or both by Annexin V-FITC and propidium iodide dual staining. Untreated cells were used as the control. The $100 \mu \mathrm{M}$ fenofibrate treatment group and the IR group significantly induced the apoptosis of CNE-2 Cells compared with the control. Critically, the results showed that the apoptosis rate were at a great extent higher in combination treatment groups than in irradiation or fenofibrate group alone and the effects were dose dependent (Figure 2A and 2B).

To further elucidate the apotosis caused by fenofibrate and irradiation, we measured the catalytic activity of caspase- 3 in KB cells. After KB cells were treated with $100 \mathrm{uM}$ fenofibrate, irradiation or both, caspase- 3 activities were increased to $112.01 \%, 149.01 \%$ and $183.81 \%$ in comparison with the internal control (Figure 2C). The 
Table 1. The Radiosensitization Activity of Fenofibrate in CNE-2 Cells

\begin{tabular}{lcccc}
\hline & $\mathrm{D}_{0}$ & $\mathrm{D}_{\mathrm{q}}$ & $\mathrm{SF}_{2}$ & $\mathrm{SER}$ \\
\hline Control & 2.26 & 2.23 & 0.76 & \\
CNE-2/ FN $50 \mu \mathrm{M}$ & 1.87 & 1.68 & 0.64 & 1.21 \\
CNE-2/FN $100 \mu \mathrm{M}$ & 1.59 & 1.06 & 0.48 & 1.42 \\
\hline
\end{tabular}

FN: fenofibrate

A
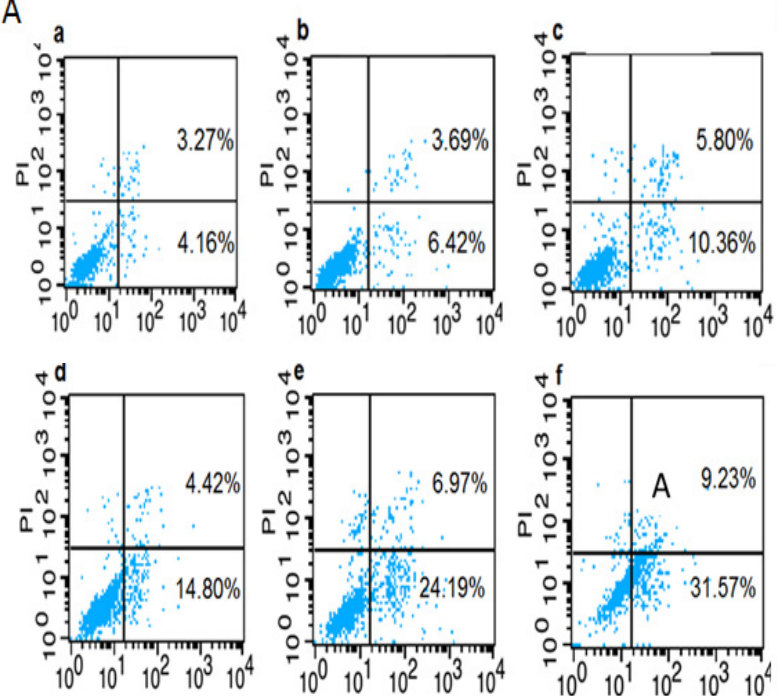

B

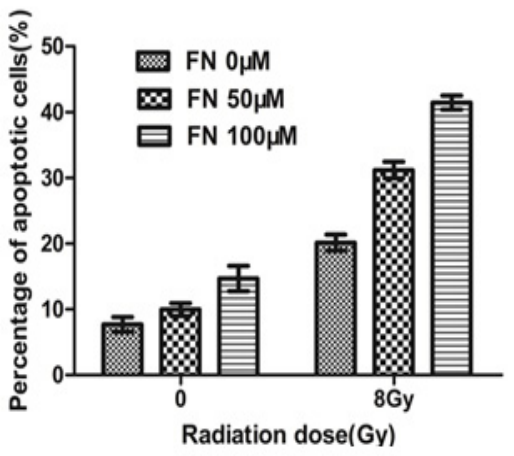

C

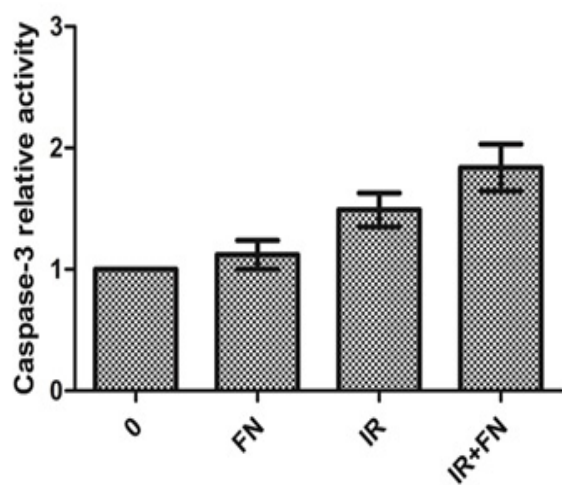

Figure 2. Flow Cytometric Analysis Showing that Fenofibrate Induced Apoptosis of CNE-2 Cells ( $A$ and B).A: a: control; b: fenofibrate $50 \mu \mathrm{M}$; c: fenofibrate 100 $\mu \mathrm{M}$; d: IR e: IR+ fenofibrate $50 \mu \mathrm{M}$; f: IR+ fenofibrate $100 \mu \mathrm{M}$. B: The apoptosis rate were at a great extent higher in combination treatment groups than in irradiation or fenofibrate group alone and the effects were dose dependent. C:The measurement of caspase-3 activity showing that After KB cells were treated with $100 \mathrm{uM}$ fenofibrate, irradiation or both, caspase-3 activities were increased to $112.01 \%, 149.01 \%$ and $183.81 \%$ in comparison with the internal control. FN: fenofibrate.
Table 2. The Radiosensitization Activity of Fenofibrate in KB Cells

\begin{tabular}{lcccr}
\hline & $\mathrm{D}_{0}$ & $\mathrm{D}_{\mathrm{q}}$ & $\mathrm{SF}_{2}$ & SER \\
\hline Control & 2.14 & 2.82 & 0.84 & \\
$\mathrm{~KB} / \mathrm{FN} 50 \mu \mathrm{M}$ & 1.8 & 2.01 & 0.7 & 1.19 \\
$\mathrm{~KB} / \mathrm{FN} 100 \mu \mathrm{M}$ & 1.63 & 0.54 & 0.38 & 1.32 \\
\hline FN: fenofibrate & & & &
\end{tabular}

A
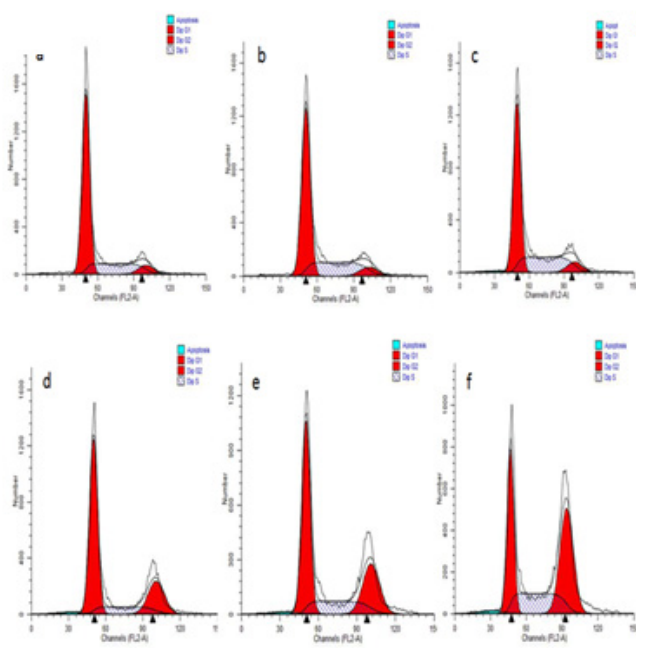

B

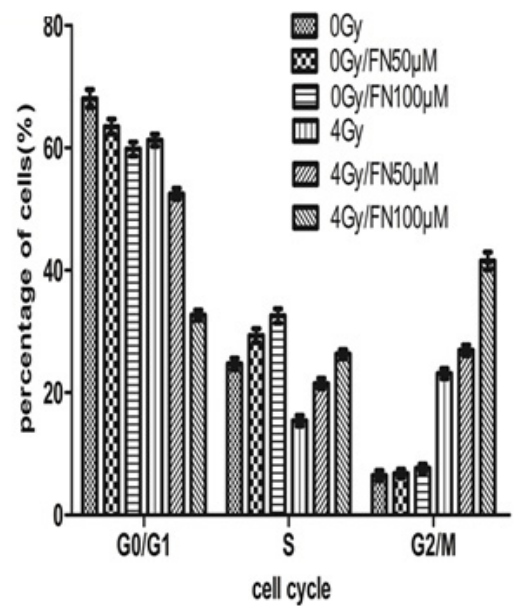

C

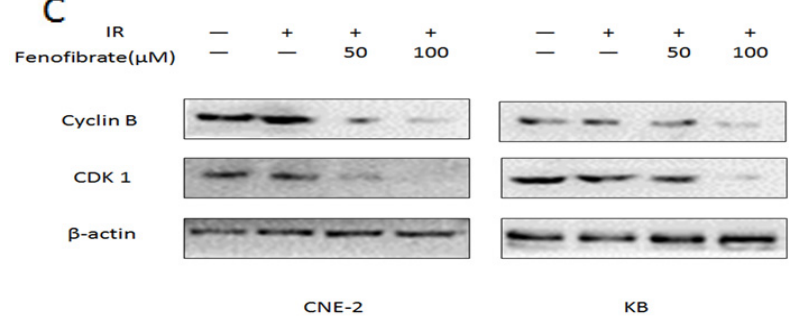

Figure 3. Flow Cytometric Analysis Showing that Fenofibrate Radiosensitize CNE-2 Cells through Inducing G2/M Checkpoint (A and B). A: a: control; b: fenofibrate $50 \mu \mathrm{M}$; c: fenofibrate $100 \mu \mathrm{M}$; d: IR e: IR+ fenofibrate $50 \mu \mathrm{M}$; f: IR+ fenofibrate $100 \mu \mathrm{M}$. B: Compared with the control or fenofibrate group, an accumulation of CNE-2 cells in the G2/M phase was noted in combination treatment groups, coupled with a decrease of the G0/G1 proportion, especially in the high dose group. C: Western blot analysis revealed that the expression of cyclinB and CDK1 proteins decreased in fenofibrate treatment cells in a dose-dependent manner. FN: fenofibrate. 
results showed that the combination group and irradiation group could significantly increase caspase-3 activity.

Fenofibrate radiosensitize HNSCC cells through inducing G2/M Checkpoint and regulating protein expression of cyclinB, CDK1

To determine the mechanisms associated with fenofibrate induced radiosensitivity of HNSCC cells, cell cycle distribution was evaluated by PI staining. Figure $3 \mathrm{~A}$ showed the relative proportion of cells at G0/G1 phase, $\mathrm{S}$ phase and G2/M phase of the cell cycle. We treated CNE-2 cells with fenofibrate for 24 hours. Compared with the control or fenofibrate group, an accumulation of CNE-2 cells in the $\mathrm{G} 2 / \mathrm{M}$ phase was noted in combination treatment groups, coupled with a decrease of the G0/G1 proportion, especially in the high dose group (Figure 3B).

To further investigate the molecular mechanisms underlying the fenofibrate inhibition of cell cycle, we examined the expression of cyclinB and CDK1 in the CNE-2 and KB cells. Western blot analysis revealed that the expression of these proteins decreased in fenofibrate treatment cells in a dose-dependent manner (Figure 3C).

Taken together, these data indicated that the fenofibratemediated increase in radiosensitivity may be partially attributed to cell cycle shifts toward the G2/M phase, which due to the decreasing expression of cyclinB and CDK1.

Fenofibrate promotes radiation sensitivity in nude mice

To determine the potential radiosensitization effect of fenofibrate on HNSCC tumor in vivo, CNE-2 tumorbearing mice were treated with a single fraction of $8 \mathrm{~Gy}$ irradiation. Mice received intraperitoneal injection of fenofibrate $(10 \mathrm{mg} / \mathrm{kg})$ for two days before the radiation. Compared to control group, no distinct difference in tumor volume was observed in fenofibrate -treated groups, while significant inhibition of tumor volume was observed in the group treated with both irradiation and fenofibrate comp ared with the group treated with irradiation alone $(p<0.05)$ (Figure 4A and 4B).

Furthermore, we analyzed the doubling time required for the tumor to grow twice in size under different treatments. Doubling time for CNE-2 tumor in control group and fenofibrate alone group was $6.44 \pm 1.04$ days and $6.69 \pm 1.33$ days, respectively. For irradiation treated group, the combination treatment extended the doubling time to $12.25 \pm 1.84$ days, compared to $8.56 \pm 1.60$ days in irradiation alone group. Intraperitoneal injection of fenofibrate enhanced response of CNE-2 xenografts to irradiation with an enhancement factor of 2.62 calculated by dividing the normalized tumor growth delay (Table 3 ).

\section{Discussion}

In the present study, we demonstrated that incubating fenofibrate decrease HNSCC cell viability and proliferation in a dose-dependent manner. In addition, increased apoptosis by fenofibrate was observed, especially when combined with irradiation. Furthermore, our study has provided evidence that fenofibrate enhanced the radiosensitivity of HNSCC cells via inducing G2/M arrest.

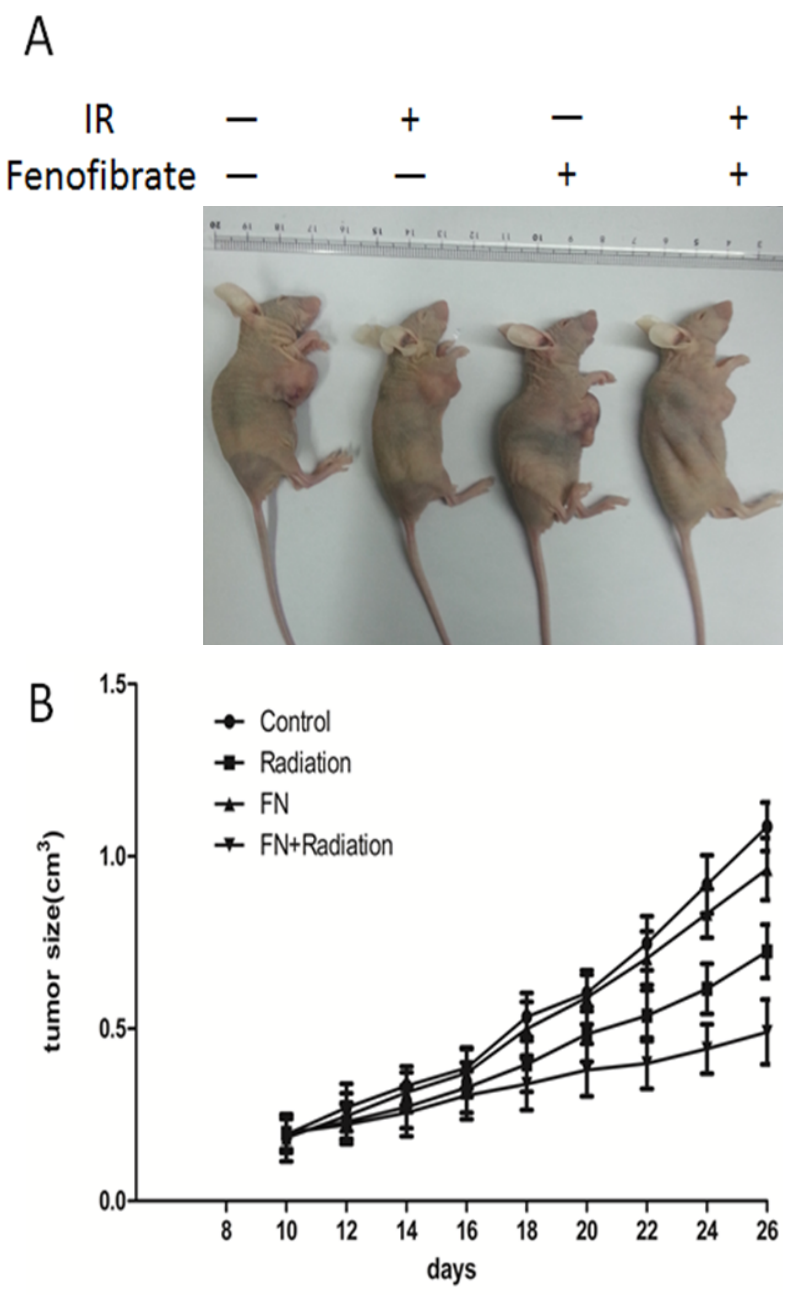

Figure 4. Fenofibrate Sensitizes HNSCC to IR in Vivo (A and B). CNE-2 xenograft bearing male BALB/c mice were divided into four treatment groups $(n=6)$ : control, fenofibrate alone, irradiation alone, or the combination of melittin and irradiation. 10 days after inoculation of CNE- 2 cells $(1 \times 106$ cells/mouse $)$, the mice were treated with a $8 \mathrm{~Gy}$-single fraction irradiation. A: Representative images of CNE-2 xenograft bearing mice. B: Measurement of tumor size. Data represent average tumor volume; error bars, SD. FN: fenofibrate.

\section{Table 3. Effect of Fenofibrate on Response of CNE-2 Xenografted Tumor to Irradiation}

\begin{tabular}{lcccc}
\hline Treatment & $\begin{array}{c}\text { Doubling time } \\
(\text { days })\end{array}$ & $\begin{array}{c}\text { Absolute growth dalay } \\
(\text { days })^{\mathrm{i}}\end{array}$ & $\begin{array}{c}\text { Normalised growth dalay } \\
(\text { days })^{\mathrm{ii}}\end{array}$ & $\begin{array}{c}\text { Enhancemet factor } \\
\text { Control }\end{array} 6^{6.44 \pm 1.04}$ \\
Fenofibrate & $6.69 \pm 1.33$ & $0.25(6.69-6.44)$ & & \\
RT & $8.56 \pm 1.60$ & $2.12(8.56-6.44)$ & & \\
RT+fenofibrate & $12.25 \pm 1.84$ & $5.81(12.25-6.44)$ & $5.56(5.81-0.25)$ & $2.62(5.56 / 2.12)$ \\
\hline
\end{tabular}

Absolute growth dalayi: The doubling tumor time of treatment group minus that of control group; Normalised growth dalayi: The time of absolute growth delay of tumor in RT combined with fenofibrate group minus that of fenofibrate group 
Besides, we observed that fenofibrate sensitized CNE-2 xenograft tumor to irradiation. Thus fenofibrate could be used as an adjuvant therapy to treat HNSCC.

It has been reported that fenofibrate plays important role in anticancer properties via diverse mechanisms in recent years (Balfour et al., 1990; Pawlik et al., 2004; Saidi et al., 2006; Grabacka et al., 2006; Urbanska et al., 2008; Zak et al., 2010; Wilk et al., 2012; Cho et al., 2013; Zhao et al., 2013; Liang et al., 2013; Watanabe et al., 2013; Li et al., 2014). But the exact relationship between fenofibrate and irradiation has not explored. Previous studies have found that fenofibrate could induce the apoptosis of cancer cells (Zak et al., 2010; Li et al., 2014) and we have also shown that fenofibrate and irradiation could induce the apoptosis of HNSCC cells by both Annexin V-FITC and propidium iodide dual staining and the catalytic activity of caspase-3. Caspase is an executer of apoptosis associated with the two signaling pathways. Regardless it is the mitochondrial or the cell death receptor pathway, they ultimately activate caspase- 3 which is essential for DNA fragmentation. Therefore, the activity of caspase- 3 is considered as an appropriate measure of cytotoxic responsiveness. Previous studies reported that many drugs induced apoptosis mainly through mitochondria/ caspase pathway in Human Gastric Cancer (Sun et al., 2013), Breast Cancer (Zhou et al., 2013), human leukemic and hepatocellular carcinoma (Banjerdpongchai et al., 2013). In this study, we found the combination group and irradiation group could significantly increase caspase-3 activity. Therefore, we suggest that Apoptosis induction was a possible mechanism for fenofibrate to increase the radiosensitivity of HNSCC cells.

Similar to apoptosis, cell cycle arrest is another index for cell growth inhibition. Katarzyna Urbanska revealed that fenofibrate contributed to the $\mathrm{G} 2 / \mathrm{M}$ phase arrest of aggressive mouse medulloblastoma cells and decrease in the $\mathrm{S}$ phase of the cell cycle (Urbanska et al., 2008). So it is rational to postulate that the mechanism of radiosensitization effect of fenofibrate on HNSCC cells may be related in the regulating of the cell cycle. In the present studies, we analyzed the changes in the cell cycle progression by using flow cytometry. The results showed that fenofibrate alone and radiation alone induced G2/M-phase arrest in the CNE-2 cells, which was further enhanced by the fenofibrate and radiation combination treatment. This result may be attributed to the radiosensitization effect of fenofibrate on HNSCC cells.

It is widely accepted that response of cells to radiation depends on the phase of they were in at irradiation. Cells in the $\mathrm{G} 2 / \mathrm{M}$ were most sensitive to irradiation and most resistant during the latter part of the $S$ phase cell cycle (Cho et al., 2013) .Therefore, it is an effective strategy to increase the sensitivity of radiotherapy through inducing G2/M arrest (Hematulin et al., 2012; Cui et al., 2012). The cell cycle involves a series of events driven by cyclins and subsequent CDKs, which further activate transcription factor proteins for cell cycle progression from one phase to another. The CDK1/cyclin B1 kinase complex plays a major role during $\mathrm{G} 2 / \mathrm{M}$ transition. A reduction in CDK1/cyclin B1 kinase activity triggers G2/M cell cycle arrest (Cui et al., 2012; Khan et al., 2011). Accumulated evidence has suggested that a lot of radiosensitizing agents worked in different cancers such as taxol (Herscher et al., 1999), oxamate (Zhai et al., 2013) through inducing G2/M arrest via downregulation of cyclinB and CDK1. In the present study, a decrease in the protein levels of cyclin B1 and CDK1 were observed in the combined treatment of fenofibrate with irradiation group. In previous studies, PPARa agonists are reported to augment TRB3 expression (Morse et al., 2009) and upregulate the gene related to proteasome biosynthesis (Cariello et al., 2005), both of which could reduce the expression of Cyclin B1. Fenofibrate is known to be a PPARa agonist, thus, it is reasonable that fenofibrate may induce G2/M arrest via reducing the activity of the CDK1/cyclin B1 kinase complex and thus increase the radiation sensitivity.

In conclusion, in this study, we demonstrated for the first time that fenofibrate could significantly promote the radiosensitivity of HNSCC cells via inducing the apoptosis and G2/M arrest via the CDK1/cyclin B1 pathway. Furthermore, we observed that fenofibrate sensitized CNE-2 xenograft tumor to irradiation. These results expand our understanding of the mechanisms of fenofibrate activity and suggest that fenofibrate is a potent radiosensitization agent for HNSCC treatment. Further studies are required to explore the other mechanisms of the radiosensitization effects of fenofibrate in HNSCC cell lines.

\section{Acknowledgements}

This study was supported by the Natural Science Foundation of China (No. 81272504), the Innovation Team (No. LJ201123-EH11), Jiangsu Provincial Science and Technology Projects BK2011854 (DA11), the Six Major Talent Peak Project of Jiangsu Province, A project funded by the priority academic program development of Jiangsu Higher Education Institution (JX10231801), grants from Key Academic Discipline of Jiangsu Province "Medical Aspects of Specific Environments" and "333" Project of Jiangsu Province BRA2012210 (RS12).

\section{References}

Akervall J, Nandalur S, Zhang J, et al (2014). A novel panel of biomarkers predicts radioresistance in patients with squamous cell carcinoma of the head and neck. Eur $J$ Cancer, 50, 570-81.

Balfour JA, McTavish D, Heel RC (1990). Fenofibrate. A review of its pharmacodynamic and pharmacokinetic properties and therapeutic use in dyslipidaemia. Drugs, 40, 260-90.

Banjerdpongchai R, Khaw-On P, Ristee C, Pompimon W (2013). 6,8-dihydroxy-7-methoxy-1-methyl-azafluorenone induces caspase-8- and -9-mediated apoptosis in human cancer cells. Asian Pac J Cancer Prev, 14, 2637-41.

Cho HJ, Oh YJ, Han SH, et al (2013). Cdk1 protein-mediated phosphorylation of receptor-associated protein 80 (RAP80) serine 677 modulates DNA damage-induced G2/M checkpoint and cell survival. J Biol Chem, 288, 3768-76.

Cui YH, Liang HJ, Zhang QQ, et al (2012). Radiosensitivity enhancement by arsenic trioxide in conjunction with hyperthermia in the EC-1 esophageal carcinoma cell line. Asian Pac J Cancer Prev, 13, 1693-7.

Cariello NF, Romach EH, Colton HM, et al (2005). Gene 
expression profiling of the PPAR-alpha agonist ciprofibrate in the cynomolgus monkey liver. Toxicol Sci, 88, 250-64.

Grabacka M, Plonka PM, Urbanska K, Reiss K (2006). Peroxisome proliferator-activated receptor alpha activation decreases metastatic potential of melanoma cells in vitro via down-regulation of Akt. Clin Cancer Res, 12, 3028-36.

Hematulin A, Meethang S, Ingkaninan K, et al (2012). Derris scandens Benth extract potentiates radioresistance of Hep2 laryngeal cancer cells. Asian Pac J Cancer Prev, 13, 1289-95.

Herscher LL, Cook J (1999). Taxanes as radiosensitizers for head and neck cancer. Curr Opin Oncol, 11, 183-6.

Joe Y, Do MH, Seo E, et al (2010). Fenofibrate antagonizes Chk2 activation by inducing Wip1 expression: implications for cell proliferation and tumorigenesis. Life Sci, 86, 716-21.

Khan M, Rasul A, Yi F, et al (2011). Jaceosidin induces p53dependent G2/M phase arrest in U87 glioblastoma cells. Asian Pac J Cancer Prev, 12, 3235-8.

Li XM, Di B, Shang YD, et al (2013). Value of postoperative radiation therapy for regional control after dissection in head and neck squamous cell carcinoma cases. Asian Pac J Cancer Prev, 14, 4273-8.

Liang H, Kowalczyk P, Junco JJ, et al (2013). Differential Effects on Lung Cancer Cell Proliferation by Agonists of Glucocorticoid and PPARalpha Receptors. Mol Carcinog, 53, 753-63.

Li T, Zhang Q, Zhang J, et al (2014). Fenofibrate induces apoptosis of triple-negative breast cancer cells via activation of NF-kappaB pathway. BMC Cancer, 14, 96.

Lindqvist A, van Zon W, Karlsson Rosenthal C, Wolthuis RM (2007). Cyclin B1-Cdk1 activation continues after centrosome separation to control mitotic progression. PLoS Biol, 5, 123.

Morse E, Selim E, Cunard R (2009). PPARalpha ligands cause lymphocyte depletion and cell cycle block and this is associated with augmented TRB 3 and reduced Cyclin B1 expression. Mol Immunol, 46, 3454-61.

Parkin DM, Bray F, Ferlay J, et al (2005). Global cancer statistics, 2002. CA Cancer J Clin, 55, 74-108.

Pawlik TM, Keyomarsi K (2004). Role of cell cycle in mediating sensitivity to radiotherapy. Int J Radiat Oncol Biol Phys, $\mathbf{5 9}, 928-42$.

Ragin CC, Modugno F, Gollin SM (2007). The epidemiology and risk factors of head and neck cancer: a focus on human papillomavirus. J Dent Res, 86, 104-14.

Saidi SA, Holland CM, Charnock-Jones DS, Smith SK (2006). In vitro and in vivo effects of the PPAR-alpha agonists fenofibrate and retinoic acid in endometrial cancer. $\mathrm{Mol}$ Cancer, $\mathbf{5}, 13$.

Sun HJ, Meng LY, Shen Y, et al (2013). S-benzyl-cysteinemediated cell cycle arrest and apoptosis involving activation of mitochondrial-dependent caspase cascade through the p53 pathway in human gastric cancer SGC-7901 cells. Asian Pac J Cancer Prev, 14, 6379-84.

Urbanska K, Pannizzo P, Grabacka M, et al (2008). Activation of PPARalpha inhibits IGF-I-mediated growth and survival responses in medulloblastoma cell lines. Int J Cancer, 123, 1015-24.

Wilk A, Urbanska K, Grabacka M, et al (2012). Fenofibrateinduced nuclear translocation of FoxO3A triggers Bimmediated apoptosis in glioblastoma cells in vitro. Cell Cycle, 11, 2660-71.

Watanabe M, Sowa Y, Yogosawa M, Sakai T (2013). Novel MEK inhibitor trametinib and other retinoblastoma gene (RB)-reactivating agents enhance efficacy of 5-fluorouracil on human colon cancer cells. Cancer Sci, 104, 687-93.

Zhang C, Yang X, Zhang Q, et al (2014). Berberine radiosensitizes human nasopharyngeal carcinoma by suppressing hypoxiainducible factor-1alpha expression. Acta Otolaryngol, 134, 185-92.

Zhao H, Zhu C, Qin C, et al (2013). Fenofibrate down-regulates the expressions of androgen receptor (AR) and AR target genes and induces oxidative stress in the prostate cancer cell line LNCaP. Biochem Biophys Res Commun, 432, 320-25.

Zak Z, Gelebart P, Lai R (2010). Fenofibrate induces effective apoptosis in mantle cell lymphoma by inhibiting the TNFalpha/NF-kappaB signaling axis. Leukemia, 24, 147686.

Zhou J, Luo YH, Wang JR, et al (2013). Gambogenic acid induction of apoptosis in a breast cancer cell line. Asian Pac J Cancer Prev, 14, 7601-5.

Zhai X, Yang Y, Wan J, et al (2013). Inhibition of LDH-A by oxamate induces $\mathrm{G} 2 / \mathrm{M}$ arrest, apoptosis and increases radiosensitivity in nasopharyngeal carcinoma cells. Oncol Rep, 30, 2983-91. 